\title{
Case Report \\ Pseudothrombocytopenia Inducing Nonindicated Platelet Transfusion after Cardiac Surgery
}

\author{
Enise Ceran $\mathbb{D D}^{1}{ }^{1}$ Christine Schlömmer ${ }^{D}{ }^{2}$ Ivonne Kröckel, ${ }^{2}$ Georg Scheriau, ${ }^{2}$ \\ Philipp Angleitner $\mathbb{D}^{1}{ }^{1}$ and Barbara Steinlechner $\mathbb{D}^{2}$ \\ ${ }^{1}$ Department of Surgery, Division of Cardiac Surgery, Medical University of Vienna, Vienna, Austria \\ ${ }^{2}$ Department of Anesthesia, Critical Care and Pain Medicine, \\ Division of Cardiothoracic and Vascular Anesthesia and Intensive Care Medicine, Medical University of Vienna, \\ Vienna, Austria
}

Correspondence should be addressed to Barbara Steinlechner; barbara.steinlechner@meduniwien.ac.at

Received 13 May 2020; Revised 17 February 2021; Accepted 22 February 2021; Published 5 March 2021

Academic Editor: Georgios D. Kotzalidis

Copyright (C) 2021 Enise Ceran et al. This is an open access article distributed under the Creative Commons Attribution License, which permits unrestricted use, distribution, and reproduction in any medium, provided the original work is properly cited.

Pseudothrombocytopenia (PTCP) is an in vitro phenomenon of low platelet count caused by the agglutination of platelets, leading to false low platelet counts in automated cell counting. Typically, ethylenediaminetetraacetic acid (EDTA) mediates this platelet clumping. PTCP has little clinical significance, but misdiagnosis may lead to unnecessary diagnostic tests and treatment. In this case report, we present a 65-year-old Caucasian female suffering from multiple complications during and after cardiac surgery. During her postoperative stay at the ICU, she was diagnosed with thrombocytopenia and an inadequate response to platelet supplementation.

\section{Case Presentation}

A 65-year-old Caucasian female was admitted to the General Hospital of Vienna with the diagnosis of a combined highgrade mitral and tricuspid valve insufficiency, persisting atrial fibrillation, and a hypertrophic nonobstructive cardiomyopathy. Surgical reconstruction of the mitral and tricuspid valve, as well as a left atrial appendage (LAA) resection, was indicated and performed. An intraoperative rupture of the left atrium led to two periods of cardiopulmonary bypass and the consecutive implantation of venoarterial extracorporeal membrane oxygenation (ECMO) due to impaired right ventricular function. After the surgical procedure, the patient was transferred to the ICU, where she required high doses of catecholamine support and continuous venovenous hemodialysis (CVVHD) due to acute renal failure. On postoperative day (POD) 7, ECMO weaning was successful, and the therapy was finally stopped. Initially, anticoagulation therapy consisted of unfractionated heparin and was switched to lowmolecular-weight heparin after ECMO explantation. On
POD 8, a complete blood count (CBC) showed a reduced platelet count of $39 \mathrm{G} / \mathrm{L}$ (Figure 1). 4Ts score for heparininduced thrombocytopenia (HIT) was 5 , which represented an intermediate probability of HIT. An enzyme-linked immunosorbent assay (ELISA) for HIT was negative (Zymutest HIA IgG, Hyphen Biomed, Neuville sur Oise, France), and a viscoelastic test of a whole blood sample (rotational thromboelastometry, ROTEM) showed no abnormalities, moreover, a procoagulant state despite the low numeric platelet count (Figure 2). Physical examination did not reveal any signs of hemorrhagic diathesis. Platelet count could not be significantly increased by the transfusion of two units of platelet concentrates. The platelet count after the first transfusion was $28 \mathrm{G} / \mathrm{L}$, and the platelet count after the second transfusion on the following day was $24 \mathrm{G} / \mathrm{L}$. On POD 10, CBC was reevaluated by using sodium citrate tubes instead of EDTA tubes, revealing a platelet count of $216 \mathrm{G} / \mathrm{L}$ (Figure 2). Since PTCP was diagnosed two days after the first low platelet count and the patient was critically ill and on continuous ECMO therapy, other causes of thrombocytopenia such as immune thrombocytopenia were not evaluated 


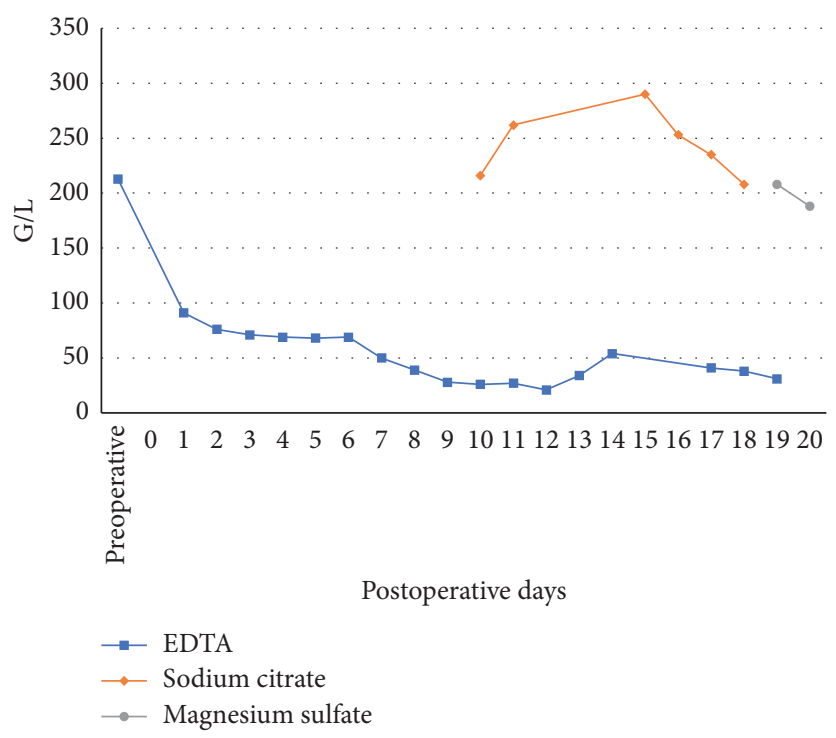

FIGURe 1: Postoperative platelet count with different blood collection tubes (EDTA, Sodium Citrate, Magensium sulfate) as shown.

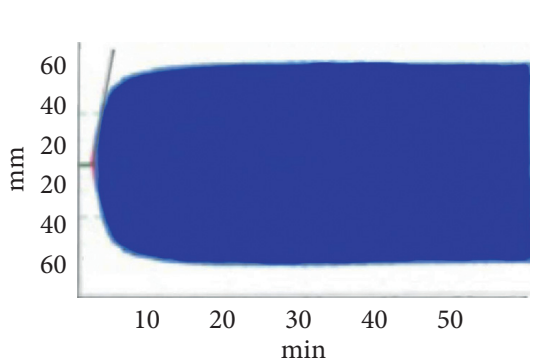

EXTEM

RT: 01:19:05

CT: $\quad 106 \mathrm{~s} \quad(38-79)$

CFT: $\quad 39 \mathrm{~s} \quad(34-159)$

A10: $\quad 75 \mathrm{~mm} \quad(43-65)$

A20: $\quad 78 \mathrm{~mm} \quad(50-71)$

MCF: $\quad 79 \mathrm{~mm} \quad(50-72)$

$\alpha: \quad 82^{\circ} \quad(63-83)$

ML: $\quad 3 \% \quad(0-15)$
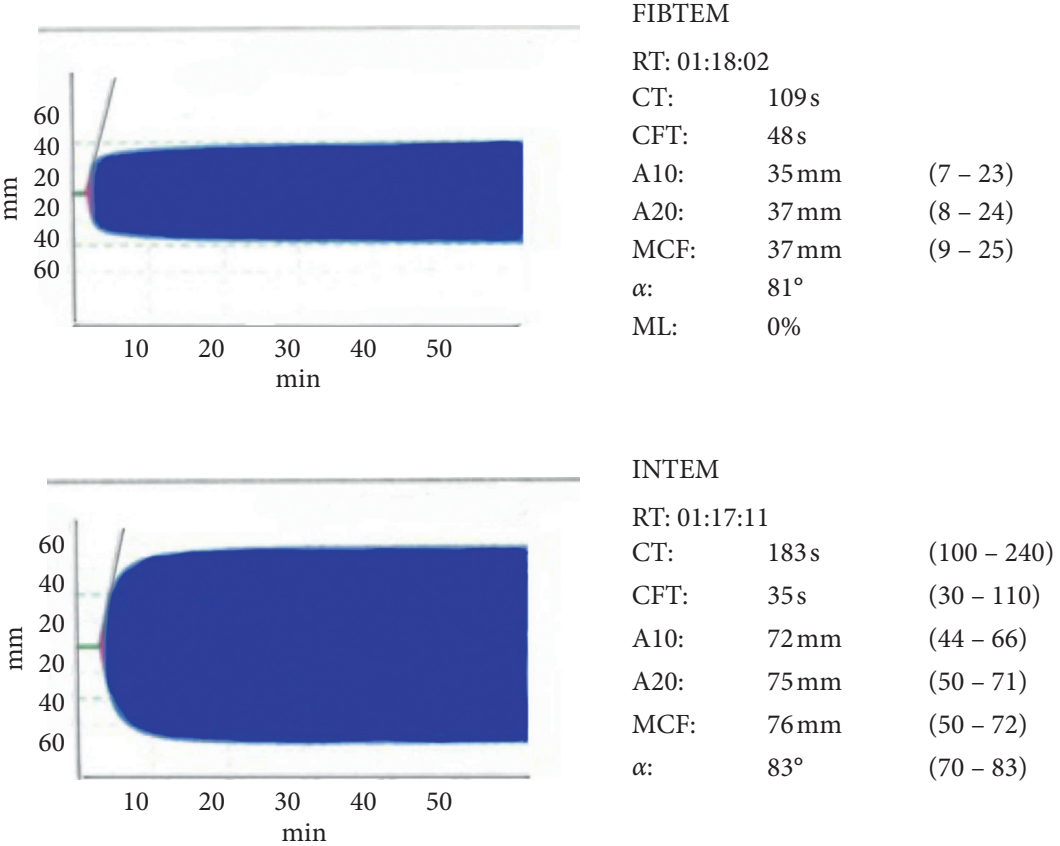

Figure 2: Rotational thromboelastometry (ROTEM) showing a procoagulant state despite the low numeric platelet count. 
further. During the patient's stay at our ICU, platelet count was also checked with a specific blood collection tube (ThromboExact, Sarstedt, Nümbrecht, Germany) confirming the results collected with the citrated blood tubes.

\section{Discussion}

Thrombocytopenia is defined as a platelet count lower than $150 \mathrm{G} / \mathrm{L}$. A platelet count lower than $100 \mathrm{G} / \mathrm{L}$ is considered to imply medium to high bleeding risk, depending on the particular platelet count [1]. The diagnosis of thrombocytopenia usually includes a repetition of the complete blood count [2-4], the white blood cell differential [5], a peripheral blood smear, and the evaluation of possible hematological abnormalities. If none of these tests are indicative for a genuine thrombocytopenia or a malignant hematological disease, PTCP can be the cause for low platelet count $[6,7]$.

PTCP is an analytical error in automated blood cell counting. Blood samples which contain autoantibodies against thrombocytes and are collected in EDTA-containing tubes can lead to platelet clumping. Agglutinated platelets are detected as larger cells by using automated counters and lead to false low results. This phenomenon was discovered early after the invention of laboratory medicine automation and was described precisely soon after [8].

The incidence of severe thrombocytopenia $(<100 \mathrm{G} / \mathrm{L})$ among the population of critically ill patients is $7.8 \%$ and therefore a very likely diagnosis. This could mislead clinicians in cases of PTCP and result in overtreatment with the administration of platelet concentrates or even more invasive procedures such as splenectomy. Other rare causes of thrombocytopenia such as HIT were found to be $0.3 \%$ in this population [9], whereas the incidence of PTCP is suggested to range from 0.09 to $0.15 \%$ in routine clinical blood samples $[10,11]$. Unfortunately, data of PTCP in a critically ill population are lacking, but given the data, one can assume that the likelihood of other more clinically relevant causes of thrombocytopenia, even if it is still very small (HIT $0.3 \%$ ), is higher than to suffer from PTCP. Therefore, clinicians are concerned with the diagnostics of the more severe forms of thrombocytopenia and are not aware about analytical errors since the repetition of the specimen often confirms the result.

As mentioned above, PTCP is induced by the crossreaction of EDTA and platelet autoantibodies which cause the agglutination of platelets in the sample [8]. This phenomenon is not only restricted to EDTA but also present with other anticoagulants such as sodium oxalate, sodium citrate, and heparin, making it more difficult to diagnose PTCP [12]. Recently, there are increasing numbers of reports portraying this multi-anticoagulant PTCP [13-15]. Additives mixed with EDTA specimens, i.e., aminoglycosides such as kanamycin and amikacin, can prevent the agglutination of platelets and dissociate platelet clumps if added after blood withdrawal and could therefore be a helping agent in diagnostics $[16,17]$. Unfortunately, there have been reports on the unreliability of these effects in certain cases of PTCP [11]. Another possible solution of the problem could be the utilization of magnesium sulfate $\left(\mathrm{MgSO}_{4}\right)$ as an anticoagulant. Before laboratory medicine automation, $\mathrm{MgSO}_{4}$ was widely used to prevent blood cell agglutination but was replaced by EDTA after the invention of automated cell counting because of the more convenient use as spraydried coat in blood collection tubes. It is now suggested to reconsider $\mathrm{MgSO}_{4}$ as an anticoagulant in diagnostics of multiple-anticoagulant PTCP [18]. By now, blood collection tubes containing $\mathrm{MgSO}_{4}$ as an anticoagulant additive are available commercially (ThromboExact, Sarstedt, Nümbrecht, Germany) and were used in this presented case to confirm the results, which were collected in citrated blood tubes.

The induction of autoantibodies reacting with EDTA and platelets is not resolved but very likely induced over a period of medical treatment. An early work on the patient population (18 patients) presenting with PTCP found that, at hospital admission, $61 \%$ of all patients had a platelet count over $100 \mathrm{G} / \mathrm{L}$, which then deteriorated over their hospital stay. Furthermore, they found that $55 \%$ of the study population were treated at an intensive care unit, $22 \%$ suffered from neoplastic diseases, and another $22 \%$ suffered from atherosclerosis-related conditions [19]. However, newer investigations could not reveal a correlation between these clinical characteristics and the development of PTCP, except for receiving medical treatment within a short period of time $[10,11]$.

The assumption of antibodies being the cause of this phenomenon was communicated early after the introduction of PTCP [8]. The detection of specific antibodies took place in the mid-90s. Platelet agglutination is induced by a variety of immunoglobulins including IgA, IgG, and IgM antibodies, which bind to glycoprotein IIb/IIIa receptors on the platelet surface [20].

There are growing numbers of reported PTCP caused by a variety of therapies and diseases. In some cases, immunotherapy with immune checkpoint inhibitors seems to be responsible for PTCP [21], as well as direct glycoprotein IIb/ IIIa antagonists such as abciximab [22]. Furthermore, there have been reports on PTCP in COVID-19 patients [23]. It seems that multiple medical treatments, diseases, and infections can induce PTCP.

It is therefore important to raise awareness throughout the clinical and scientific societies to consider PTCP as differential diagnosis early after the onset of low platelet counts without clinical symptoms of hemorrhagic diathesis. With cases of multi-anticoagulant PTCP, it is getting more difficult to detect false low platelet counts, and appropriate diagnostic facilities (peripheral blood smear, ThromboExact blood tubes) need to be available for correct diagnosis. Considering these challenges, it can be difficult in clinical settings to thoroughly evaluate causes of thrombocytopenia, but one should consequently pursue the process of finding the right diagnosis since the clinical consequences of overtreatment can be dramatic, i.e., splenectomy. In this case report, the challenge of finding the right diagnosis was the complexity of the case. Thrombocytopenia is not uncommon after cardiac surgery and on ECMO therapy. A bleeding tendency is often present because of deranged coagulation factors and mass transfusions. Since our department is very 
skilled and trained in the management and diagnosis of bleeding disorders, our patient was diagnosed with PTCP shortly after the onset of severe thrombocytopenia (two days after first severe low platelet counts). Further invasive treatment could be averted from the patient.

\section{Conflicts of Interest}

The authors declare that they have no conflicts of interest.

\section{References}

[1] S. Vanderschueren, A. De Weerdt, M. Malbrain et al., "Thrombocytopenia and prognosis in intensive care," Critical Care Medicine, vol. 28, no. 6, pp. 1871-1876, 2000.

[2] M. L. Brigden, N. E. Page, and C. Graydon, "Evaluation of the sysmex NE-8000:automated hematology analyzer in a highvolume outpatient laboratory," American Journal of Clinical Pathology, vol. 100, no. 6, pp. 618-625, 1993.

[3] H. Inoue, "Overview of automated hematology analyzer XE2100,”, 2000.

[4] K. Ruzicka, M. Veitl, R. Thalhammer-Scherrer, and I. Schwarzinger, "The new hematology analyzer sysmex XE2100," Archives of Pathology \& Laboratory Medicine, vol. 125, no. 3, pp. 391-396, 200.

[5] M. Zandecki, F. Genevieve, J. Gerard, and A. Godon, "Spurious counts and spurious results on haematology analysers: a review. part II: white blood cells, red blood cells, haemoglobin, red cell indices and reticulocytes," Clinical and Laboratory Haematology, vol. 29, no. 1, pp. 21-41, 2007.

[6] M. Nagler, P. Keller, D. Siegrist, and L. Alberio, "A case of EDTA-dependent pseudothrombocytopenia: simple recognition of an underdiagnosed and misleading phenomenon," BMC Clinical Pathology, vol. 14, no. 1, p. 19, 2014.

[7] E. Gowland, H. Kay, J. C. Spillman, and J. R. Williamson, "Agglutination of platelets by a serum factor in the presence of EDTA," Journal of Clinical Pathology, vol. 22, no. 4, pp. 460-464, 1969.

[8] D. P. Shreiner and W. R. Bell, "Pseudothrombocytopenia: manifestation of a new type of platelet agglutinin," Blood, vol. 42, no. 4, pp. 541-549, 1973.

[9] D. R. Williamson, M. Albert, D. Heels-Ansdell et al., "Thrombocytopenia in critically ill patients receiving thromboprophylaxis," Chest, vol. 144, no. 4, pp. 1207-1215, 2013.

[10] J. García Suárez, J. L. Merino, M. Rodríguez, A. Velasco, and M. C. Moreno, "Pseudothrombocytopenia: incidence, causes and methods of detection," Sangre (Barc), vol. 36, no. 3, pp. 197-200, 1991.

[11] J. Lin, Y. Luo, S. Yao et al., "Discovery and correction of spurious low platelet counts due to EDTA-dependent pseudothrombocytopenia," Journal of Clinical Laboratory Analysis, vol. 29, no. 5, pp. 419-426, 2015.

[12] H. Schrezenmeier, H. Müller, E. Gunsilius, H. Heimpel, and E. Seifried, "Anticoagulant-induced pseudothrombocytopenia and pseudoleucocytosis," Thrombosis and Haemostasis, vol. 73, no. 03, pp. 506-513, 1995.

[13] F. Kovacs, M. Varga, Z. Pataki, and E. Rigo, "Pseudothrombocytopenia with multiple anticoagulant sample collection tubes," Interventional Medicine and Applied Science, vol. 8, no. 4, pp. 181-183, 2016.

[14] L. Zhong, J. Chadha, and A. Ameri, "A curious case of pseudothrombocytopenia due to in vitro agglutination," Case
Reports in Hematology, vol. 2020, Article ID 6236350, 3 pages, 2020.

[15] H. özlem, "Management of a case with EDTA and citrate dependent pseudothrombocytopenia in the laboratory," International Journal of Medical Biochemistry, vol. 4, no. 1, pp. 56-59, 2020.

[16] S. Sakurai, I. Shiojima, T. Tanigawa, and K. Nakahara, "Aminoglycosides prevent and dissociate the aggregation of platelets in patients with EDTA-dependent pseudothrombocytopenia," British Journal of Haematology, vol. 99, no. 4, pp. 817-823, 1997.

[17] X. Zhou, X. Wu, W. Deng, J. Li, and W. Luo, "Amikacin can be added to blood to reduce the fall in platelet count," American Journal of Clinical Pathology, vol. 136, no. 4, pp. 646-652, 2011.

[18] S. Mannuß, P. Schuff-Werner, K. Dreißiger, and C. Burstein, "Inhibition of agonist-induced platelet aggregation by magnesium sulfate warrants its use as an alternative in vitro anticoagulant in pseudothrombocytopenia," Platelets, vol. 31, no. 5, pp. 680-684, 2020.

[19] N. Berkman, Y. Michaeli, R. Or, and A. Eldor, "Edta-dependent pseudothrombocytopenia: a clinical study of 18 patients and a review of the literature," American Journal of Hematology, vol. 36, no. 3, pp. 195-201, 1991.

[20] A. Casonato, A. Bertomoro, E. Pontara, D. Dannhauser, A. R. Lazzaro, and A. Girolami, "EDTA dependent pseudothrombocytopenia caused by antibodies against the cytoadhesive receptor of platelet gpIIB-IIIA," Journal of Clinical Pathology, vol. 47, no. 7, pp. 625-630, 1994.

[21] K. Krukowska, R. Kieszko, K. Kurek, I. Chmielewska, P. Krawczyk, and J. Milanowski, "An episode of pseudothrombocytopenia during pembrolizumab therapy in NSCLC patient," Case Reports in Oncological Medicine, vol. 2020, Article ID 4196178, 4 pages, 2020.

[22] H. Stiegler, Y. Fischer, S. Steiner, B. E. Strauer, and H. Reinauer, "Sudden onset of EDTA-dependent pseudothrombocytopenia after therapy with the glycoprotein IIb/IIIa antagonist c7E3 Fab," Annals of Hematology, vol. 79, no. 3, pp. 161-164, 2000.

[23] H. Li, B. Wang, L. Ning, Y. Luo, and S. Xiang, "Transient appearance of EDTA dependent pseudothrombocytopenia in a patient with 2019 novel coronavirus pneumonia," Platelets, vol. 31, no. 6, pp. 825-826, 2020. 